\title{
Summary of the TeV33 Working Group
}

\author{
P.P. Bagley, ${ }^{1}$ F.M. Bieniosek, ${ }^{1}$ P. Colestock, ${ }^{1}$ A. Gerasimov, ${ }^{1}$ G.P. Jackson, ${ }^{1}$ J.A. Johnstone, ${ }^{1}$ \\ J.A. MacLachlan, ${ }^{1}$ J.P. Marriner, ${ }^{1}$ C.S. Mishra, ${ }^{1}$ S.M. Pruss, ${ }^{1}$ S. Shukla, ${ }^{1}$ R.H. Siemann, ${ }^{2}$ W. Wan ${ }^{3}$ \\ ${ }^{1}$ Fermilab, P.O. Box 500, Batavia, IL 60510 \\ ${ }^{2}$ Stanford Linear Accelerator Center, Stanford University, Stanford, CA 94309 \\ ${ }^{3}$ University of Colorado, Boulder, CO 80309
}

\begin{abstract}
This summary of the TeV33 working group at Snowmass reports on work in the areas of Tevatron store parameters, the beam-beam interaction, Main Injector intensity (slip stacking), antiproton production, and electron cooling.
\end{abstract}

\section{OVERVIEW OF TEV33}

The goal of the TeV33 project is to develop a plan for the Fermilab Tevatron Collider beyond the year 2000 when the Main Injector project will be finished and collider Run II is expected to be well underway.

The TeV2000 committee report [1] suggests a goal of 30 $\mathrm{fb}^{-1}$ of proton-antiproton collisions at $2 \mathrm{TeV}$ in the center of mass system by the year 2006. The physics potential of such a data sample is summarized in the committee's report and is described in more detail in the report of the TeV2000 Study Group [2].

The goal of obtaining a sample of $30 \mathrm{fb}^{-1}$ of protonantiproton collisions is widely believed to be technically feasible, but it is uncertain whether it can be achieved within the likely fiscal and schedule constraints. An upgrade to a higher energy or a higher luminosity proton-proton collider is also technically possible but, based on performance, cost, and schedule considerations, is judged to be an unrealistic competitor for the Large Hadron Collider (LHC).

The purpose of the TeV33 group and, in particular, the Snowmass study group, is to develop a realistic plan for TeV33 that can form the basis for future decisions. The TeV33 project assumes the successful completion of the Main Injector project and other upgrade projects that are associated with Run II. Some of the important elements of the Run II upgrade are:

1. Main Injector with both physical and dynamic apertures greatly exceeding those available with the existing Main Ring.

2. Antiproton recovery, including the construction of a "Recycler" ring in the Main Injector tunnel.

3. Antiproton source improvements in targeting and beam cooling.

While the details of the TeV33 plan are not well defined, many of the major considerations are already clear. These are:

1. The antiproton production rate must be improved by about a factor of 5 .
2. The antiproton cooling must be improved to accommodate the increased flux. The current plan is to use

a. 4-8 GHz stochastic cooling in the existing Debuncher and Accumulator Rings, and

b. electron cooling in the Recycler Ring.

3. The existing detector technology (with modest upgrades) must be capable of analyzing the collisions for rare processes. The important considerations for the accelerator are:

a. Limiting the number of interactions per crossing to a sufficiently small value by using roughly 100 bunches in the Tevatron,

b. Reducing the peak luminosity (luminosity leveling) to further reduce the number of interactions per crossing, and

c. Maintaining low backgrounds.

4. The proton and antiproton beams must be kept well separated in the Tevatron except at the desired collision points to avoid the adverse effects of the beam-beam interaction. Obtaining adequate separation becomes increasingly difficult as the number of bunches is increased to 100 or more bunches.

At Snowmass we developed some of the aspects of the TeV33 plan, but not all the important issues were covered. This paper is intended to report the work completed in conjunction with Snowmass study, and is not a balanced presentation of the current thinking on all the important elements of the TeV33 project.

\section{ELEMENTS OF TEV33}

\section{A. Tevatron Store Parameters}

The performance of a TeV33 scenario is determined by the average production rate of events that can be analyzed to study interesting physics processes. The study described below considered various initial store parameters and calculated the dependence of the integrated luminosity on several possible choices of parameters. The more delicate question of whether the events produced could be analyzed by a given detector was not considered. 


\section{The Luminosity Calculation}

It is straight-forward to calculate the luminosity $(L)$ by integrating the beam phase-space distributions. A relatively compact expression can be obtained when the distributions are assumed to be gaussian in transverse and longitudinal positions. In this case the luminosity per interaction region is given by

$$
\begin{aligned}
\mathcal{L} & =\frac{f_{0} B N_{p} N_{\bar{p}}}{4 \pi \sigma_{x}^{*} \sigma_{y}^{*}} \int_{-\infty}^{\infty}\left\{\frac{1}{\sqrt{\pi} \sigma_{s}\left(1+s^{2} / \beta^{* 2}\right)} \exp \left(-s^{2} / \sigma_{s}^{2}\right)\right. \\
& \left.\times \exp \left[\frac{-s^{2}}{1+s^{2} / \beta^{* 2}}\left(\frac{\theta_{x}^{2}}{\sigma_{x}^{* 2}}+\frac{\theta_{y}^{2}}{\sigma_{y}^{* 2}}\right)\right]\right\} d s
\end{aligned}
$$

where $f_{0}$ is the revolution frequency, $B$ is the number of bunches, $N_{p}\left(N_{\bar{p}}\right)$ is the number of protons (antiprotons) per bunch, $\sigma_{s}$ is the rms bunch length of either beam, and $\theta_{x}$ and $\theta_{y}$ are the crossing half-angles. The beta function is assumed to achieve a minimum value of $\beta^{*}$ at $s=0$, where the average beam size is $\sigma_{x}^{* 2}=\left(\sigma_{x p}^{* 2}+\sigma_{x \bar{p}}^{* 2}\right) / 2$ (and similarly for the $y$ plane). The luminosity formula can also be rewritten as

$$
\mathcal{L}=\frac{3 \gamma_{r} f_{0}}{\beta^{*}}\left(B N_{\bar{p}}\right)\left(\frac{N_{p}}{\varepsilon_{p}}\right) \frac{F\left[\frac{\beta^{*}}{\sigma_{s}}, \frac{\gamma_{r} \sigma_{s}^{2}}{\beta^{*}}\left(\frac{\theta_{x}^{2}}{\varepsilon_{x}}+\frac{\theta_{y}^{2}}{\varepsilon_{y}}\right)\right]}{\left(1+\varepsilon_{\bar{p}} / \varepsilon_{p}\right)}
$$

where $\gamma_{r}=E / m c^{2}$ is the relativistic energy factor, $F \leq 1$ is a formfactor that accounts for the depth of focus (hour glass) and crossing angle effects on the luminosity caused by non-zero bunch lengths, and $\varepsilon_{p}\left(\varepsilon_{\bar{p}}\right)$ is the proton (antiproton) beam transverse emittance*, which is defined to be $\varepsilon=6 \pi \gamma_{r} \sigma^{2} / \beta$ for a bunch with a gaussian distribution where the dispersion is zero. The bunch lengths depend on the longitudinal emittance and the rf voltage, but the luminosity depends only on the bunch length. The first quantity in parenthesis in Eqn. 2 is the total number of antiprotons. Since the production of antiprotons is both technically challenging and expensive, the availability of antiprotons is a major limitation in the maximum luminosity that can be produced. The second quantity in parenthesis may be limited by the tune shift produced by the collision points at the detectors. The formula for the linear beam-beam tune shift of the antiprotons for collisions with no crossing angle is:

$$
\begin{aligned}
\Delta v & =6 \frac{r_{p}}{4 \pi} n_{c} \frac{N_{p}}{\varepsilon_{p}} \\
& =0.0073\left(\pi \mathrm{mm}-\mathrm{mrad} / 10^{10}\right) n_{c} \frac{N_{p}}{\varepsilon_{p}}
\end{aligned}
$$

\footnotetext{
*The emittances referred to in sections A and B are the phase space area containing $95 \%$ of the beam and normalized by the beam energy.
}

where $r_{p}$ is the classical proton radius $\left(1.535 \times 10^{-18} \mathrm{~m}\right), N_{p}$ is the number of protons per bunch, and $n_{c}$ is the number of interaction points.

Operating experience in the Tevatron with 6 bunches and no crossing angle suggests that the maximum tolerable beambeam tune shift lies in the range 0.02 to 0.025 . In this section, we only considered cases with $N_{p} / \varepsilon_{p}$ fixed at $1.35 \times 10^{10} / \pi \mathrm{mm}^{-1} \mathrm{mrad}^{-1}$, i.e., $\Delta v=0.01$ per interaction point although a more complete analysis would include the effects of the crossing angle at the collision point and of the long-range (parasitic) crossings. The antiproton $N_{\bar{p}} / \varepsilon_{\bar{p}}$ is also limited although the limit on antiproton production has traditionally been the more important limit to antiproton intensity.

For the purposes of comparison a nominal parameter set was chosen. The nominal parameter set is shown in Table I.

Table I. Nominal TeV33 parameters compared with Run Ib.

\begin{tabular}{lcc}
\hline \hline Parameter & Run Ib & TeV33 \\
\hline$N_{p}$ & $23 \times 10^{10}$ & $27 \times 10^{10}$ \\
$N_{\bar{p}}$ & $5.5 \times 10^{10}$ & $\leq 27 \times 10^{10}$ \\
$\varepsilon_{p}(\mathrm{~mm}-\mathrm{mrad})$ & $23 \pi$ & $20 \pi$ \\
$\varepsilon_{\bar{p}}(\mathrm{~mm}-\mathrm{mrad})$ & $13 \pi$ & $20 \pi$ \\
$\varepsilon_{p}^{L}(\mathrm{eV}-\mathrm{sec})$ & 3.8 & 3 \\
$\varepsilon_{\bar{p}}^{L}(\mathrm{eV}-\mathrm{sec})$ & 3.5 & 3 \\
$\mathrm{rf}$ voltage $(\mathrm{MV})$ & 1 & 1 \\
$N_{b}$ & 6 & 100 \\
$\beta *(\mathrm{~m})$ & 0.35 & 0.35 \\
$\theta_{x}=\theta_{y}(\mu \mathrm{rad})$ & 0 & \pm 100 \\
Energy $(\mathrm{GeV})$ & 900 & 1000 \\
$\mathcal{L}\left(\mathrm{cm}^{-2} \mathrm{sec}^{-1}\right)$ & $1.62 \times 10^{31}$ & $\leq 1.16 \times 10^{33}$ \\
\hline \hline
\end{tabular}

These nominal $\mathrm{TeV} 33$ parameters are arbitrary, but the proton intensity per bunch and emittances are close to the numbers already achieved in Run Ib.

\section{Evolution of a Store}

To examine the behavior of a store with given initial conditions, a store lifetime simulation program [3] was run for a number of antiproton intensities. The luminosity versus time for the nominal store parameters is shown in Figure 1.

An important feature of Figure 1 is that the luminosity drops sharply in the first few hours of the store. Since stores are expected to last at least a few hours, the luminosity lifetime has an important effect on the integrated luminosity. Factors which contribute to the luminosity lifetime and which are included in the simulation are:

1. Loss of protons and antiprotons through collisions.

2. Longitudinal and transverse emittance growth caused by intrabeam scattering.

3. Beam loss and emittance growth from collisions with the residual gas.

Probably the most important omission in the simulation is the emittance growth arising from non-linear dynamics. Our operational experience indicates that the effect is relatively small when the Tevatron betatron tune, orbit, and other operating parameters are well adjusted. 


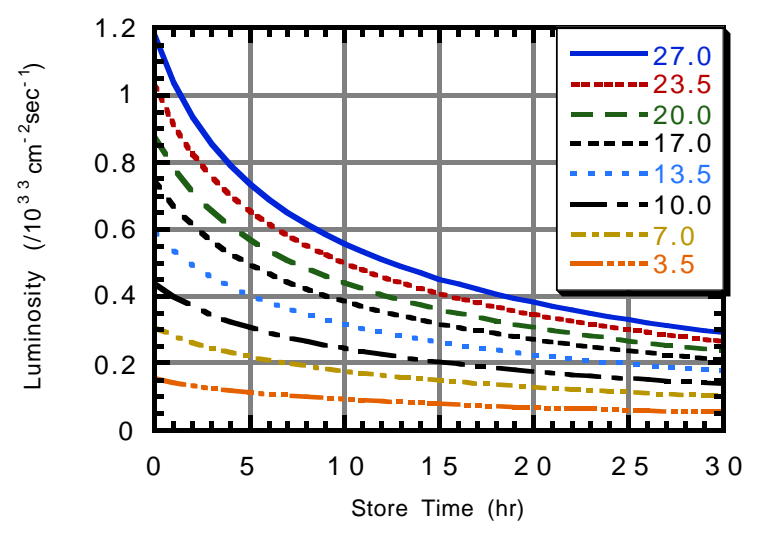

Figure 1. Luminosity versus time in a store for the initial store parameters given in Table I except for the varying antiproton intensities indicated in the legend.

The first factor affecting lifetime-the loss of particles through collisions-is a result of having produced a high luminosity. The effect of intrabeam scattering is also quite large. It is affected by beam intensity (higher bunch intensities increase the scattering rate) and beam emittance (smaller emittances generally increase the scattering rate). The residual gas effects are relatively unimportant compared to the first two. Factors that increase the initial luminosity in many cases decrease the luminosity lifetime, so the integrated luminosity is much less sensitive to changes in parameters than might be thought on a naive basis.

The rapid decrease in luminosity is not accompanied by a corresponding decrease in antiproton intensity, however. For the stores shown in Figure 1, the fraction of antiprotons remaining after a $30 \mathrm{hr}$ store ranges between 63 and $71 \%$, depending on the initial luminosity. The goal of antiproton recycling is to recapture the bulk of these antiprotons for reinjection into the Tevatron. It is expected that a Recycler Ring will be built to accomplish this goal by the time the Tevatron returns to collider operations in 1999.

If the recycling process were $100 \%$ efficient, the integrated luminosity would depend entirely on the antiproton production rate for a wide range of initial store parameters. The actual efficiency is not yet known (and will probably depend on the antiproton beam emittances at the end of a store). This study assumes that the recapture efficiency times the overall transfer efficiency to the colliding beam state in the Tevatron is $70 \%$. With this simple assumption, it is possible to compute the number of antiprotons required to re-establish the same initial antiproton intensity (in a new store). If it is further assumed that the antiproton production rate times the overall transfer efficiency to the colliding beam condition in the Tevatron is $0.8 \times 10^{12} / \mathrm{hr}$, the number of antiprotons available as a function of time can be computed. When the number of antiprotons required becomes equal to the number available, it is possible to initiate a new store. These quantities are shown graphically in Figure 2.

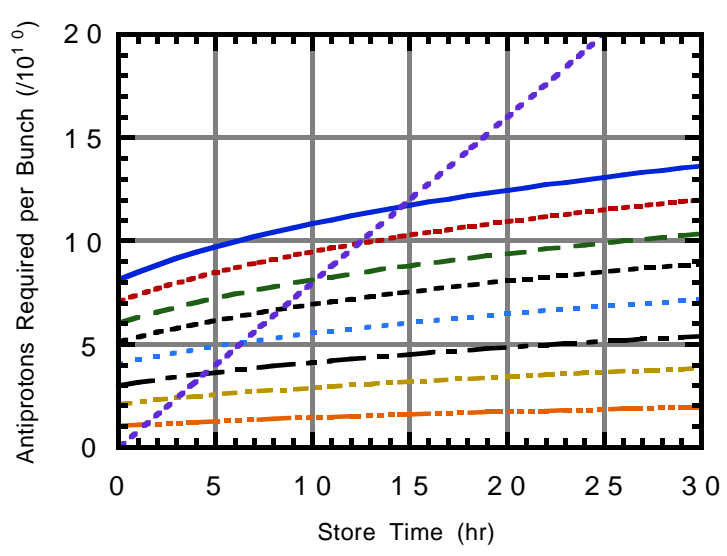

Figure 2. Number of antiprotons per bunch required versus store time for the initial parameters given in Table I but with varying antiproton intensities. The straight line from the origin shows the number of antiprotons produced per colliding bunch as a function of time assuming a stacking rate times transfer efficiency of $0.8 \times 10^{12} / \mathrm{hr}$. The initial antiproton intensities are the same as shown in the legend in Figure 1.

For a given initial antiproton intensity, the optimum store length may be determined by plotting the integrated luminosity versus the time between stores as shown in Figure 3 for the nominal parameters in Table $\mathrm{I}$ and an initial antiproton bunch intensity of $27 \times 10^{10}$. The time that is required to establish the colliding beams (shot setup time) is assumed to be 1 hour, so the store integration starts at that time. It is also assumed that no antiprotons are collected during shot setup time. The average luminosity is given by the slope of the line from the origin to any point of the curve. Provided that sufficient antiprotons were available, the highest slope determines the optimum store time: in this case it is about 5.5 hours. However, from Figure 2, the time required to replenish the antiprotons in a store is $\mathbf{1 5 . 6}$ hours-considerably longer than the optimum. This time is indicated by the marker in Figure 3. The time to replenish the antiprotons would be less than $5.5 \mathrm{hr}$ if, for example, the recycling efficiency were $94 \%$, the stacking rate were $2.1 \times 10^{12}$ antiprotons/hr, or the initial antiproton bunch intensity were less than about $11 \times 10^{10}$. Repeating this analysis with smaller values for the initial antiproton intensity, we find that $N_{\bar{p}}=27 \times 10^{10}$ with a time between stores of $15.6 \mathrm{hr}$ yields the maximum average luminosity.

\section{Choice of Initial Store Parameters}

We want to consider the integrated luminosity obtained by varying some of the nominal parameters in Table I. In order to motivate the studies performed, we will summarize some of the factors affecting the integrated luminosity. These factors are:

1. Proton intensity: The luminosity is directly proportional to the proton intensity. However, the antiproton beam- 
beam tune shift limit may constrain the maximum value of $N / \varepsilon$. The injector chain also limits the values of $N$ and $\varepsilon$ that are accessible.

2. Antiproton intensity: The luminosity is directly proportional to the antiproton intensity. The proton beam-beam tune shift limit constrains the maximum value of $N / \varepsilon$ if the antiproton production rate is high.

3. Number of bunches: For a fixed total number of antiprotons, the luminosity does not depend on the number of bunches. However, more bunches reduce the number of interactions per crossing, an important parameter for the particle physics detectors. If the antiproton intensity is limited not by the production rate, but the beam-beam tune shift limit, it is desirable to increase the number of bunches. More bunches result in more long-range beam-beam interactions which appear to cause increasingly severe problems as the bunch spacing is decreased. The trigger upgrades for the major detectors (CDF and D0) assume a minimum bunch spacing of $7 \mathrm{rf}$ buckets.

4. Transverse emittance: Smaller emittance decreases the beam size and increases the luminosity but the effect is partially offset by the decrease in the form factor. Smaller transverse emittance may require smaller bunch intensities to avoid excessive beam-beam effects at the collision points. Smaller transverse emittances are generally beneficial for the long-range beam-beam interactions. Smaller emittances increase the intrabeam scattering rate, but this effect is offset by the smaller intensities if $N / \varepsilon$ is kept constant.

5. Antiproton emittance: The antiproton emittance can be smaller than the proton emittance if the antiproton intensity is sufficiently low that the protons are not affected.

6. Bunch length: Shorter bunch lengths increase the luminosity through the hour glass effect and the crossing angle effect. The bunch length may be reduced by smaller longitudinal emittances and higher rf voltage.

7. Beta-star: A lower $\beta^{*}$ generally results in increased luminosity. However, the gain in luminosity is partially offset by the decrease in the form-factor. Furthermore, it appears that it would be difficult to reduce $\beta^{*}$ below 25 $\mathrm{cm}$ with the current low beta insertion.

8. Crossing angle: A crossing angle reduces the luminosity but appears to be necessary to avoid large beam-beam interaction effects from the collisions nearest the interaction regions. The form-factor $F$ in Eqn. 2 increases with larger emittance, larger $\beta^{*}$ and shorter bunch lengths.

9. Intrabeam scattering: The intrabeam scattering rate increases with increasing intensity and generally increases with decreasing emittances. These factors are the same factors that make the initial luminosity higher. Thus, the integrated luminosity is less sensitive to changes in these parameters than is the initial luminosity (or store lifetime).



Figure 3. Integrated luminosity for a store with the initial parameters listed in Table I and an initial antiproton intensity $N_{\bar{p}}=27 \times 10^{10}$. The maximum average integrated luminosity is obtained after $5.5 \mathrm{hr}$, but $15.6 \mathrm{hr}$ (marker) are required to stack enough antiprotons for a new store.

10. Antiproton lifetime: The antiproton lifetime is dominated by the loss through collisions. The only real solution is to produce antiprotons at higher rates.

Given this understanding of the relevant parameters, the following variations on the nominal parameter set were considered:

1. A smaller transverse emittance ( $10 \pi \mathrm{mm}$-mrad). Keeping the beam-beam tune shift constant, the maximum intensity according to Eqn. 3 is $13.5 \times 10^{10}$ per bunch.

2. A larger transverse emittance $(30 \pi \mathrm{mm}-\mathrm{mrad})$. The maximum intensity is $40 \times 10^{10}$ per bunch.

3. A smaller longitudinal emittance $(0.5 \mathrm{eV}$-sec $)$.

4. Higher $\mathrm{rf}$ voltage at 4 times the present frequency (15 $\mathrm{MV}$ at $212 \mathrm{MHz}$ ) with the longitudinal emittance at the nominal $3.0 \mathrm{eV}$-sec.

Figure 4 compares the average luminosity for each of these cases. The time between stores is determined by the length of time required to accumulate the antiprotons plus a one-hour shot setup time although, for low antiproton intensities, a somewhat greater average luminosity would be obtained with longer stores. It is striking that the integrated luminosities are rather similar in all cases. Probably the most important reason for the similarity is that the same antiproton economics constrain all scenarios. The problem with the low transverse emittance case seems to be two-fold: small emittances decrease the form factor in Eqn. 2 and the antiproton single bunch intensity is limited to a low initial value (compared to the other cases) by the proton beam-beam tune shift. The larger emittance case $(30 \pi \mathrm{mm}-\mathrm{mrad})$ is slightly more favorable, but it is doubtful whether such a large emittance could be accommodated in the Tevatron. Despite the relatively naive assumptions, we tentatively conclude that the integrated luminosity is maximized with the largest transverse emittance consistent with aperture and beam-beam force considerations. 


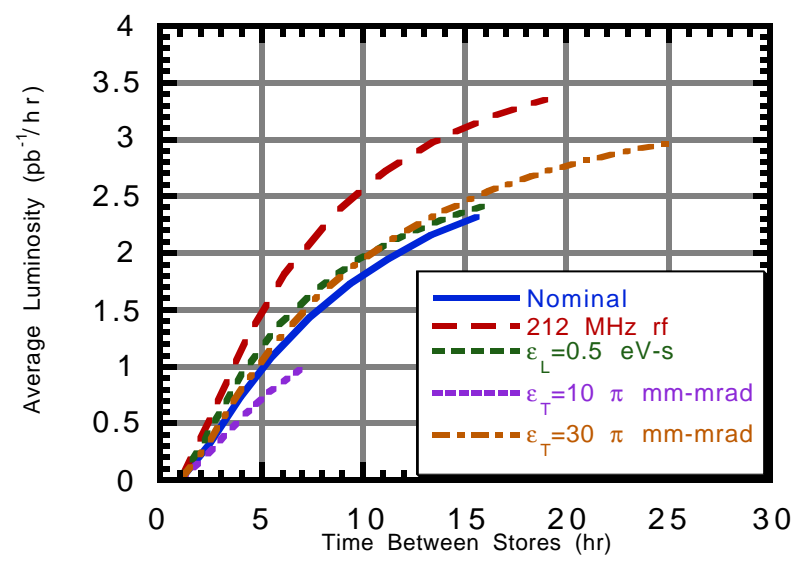

Figure 4. Average Luminosity, defined as the total integrated luminosity divided by the time since shot setup began. The time between stores is the shot setup time (assumed to be $1 \mathrm{hr}$ ) plus the time required to accumulate the necessary antiprotons.

Shorter bunch lengths, on the other hand, appear to be attractive. These small bunch lengths can be achieved by small longitudinal emittance bunches or with greater longitudinal focusing (the focusing is proportional to $\mathrm{rf}$ voltage times the frequency). The high intensity $0.5 \mathrm{eV}-\mathrm{sec}$ bunches can not be achieved with the current injector chain. Modest improvements (to about $2.0 \mathrm{eV}$-sec) are expected from the Main Injector. Further progress (to $0.5 \mathrm{eV}$-sec) might be achieved with electron cooling. Higher rf voltage makes the bunch lengths shorter while leaving the emittance unchanged. The rf upgrade seems to work better than a low initial emittance, especially for long stores.

It is interesting to compare the emittance growth for the of upgrade, the small initial longitudinal emittance, and the nominal case. The growth of longitudinal and transverse emittances are shown in Figure 5 and Figure 6. The rf upgrade increases the momentum spread of the beam and also the peak current. For longitudinal intrabeam scattering, the effect of the momentum spread is more important, and the intrabeam scattering growth is greatly reduced. The opposite is true for transverse intrabeam scattering, where the rf upgrade has the fastest heating rate of any of the scenarios considered. The very small initial longitudinal emittance $(0.5 \mathrm{eV}$-sec) case generally lies between the other two cases.

From this study, we tentatively conclude that the integrated luminosity increases monotonically with antiproton intensity. Small transverse emittance did not seem to be particularly attractive. However, this conclusion is tempered by the use of an unrealistic model of the constraints on proton emittance and intensity that are imposed by the beam-beam interaction. Smaller longitudinal emittance appears to be helpful. Shorter bunch lengths, which could be obtained by an if upgrade, appear attractive, but it is not clear that the gain would be worth the substantial cost.

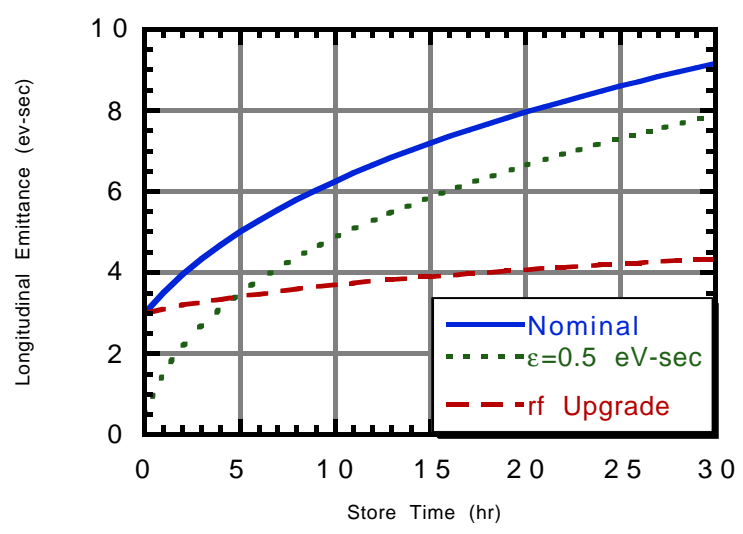

Figure 5. The time dependence of the longitudinal emittance of the stores described in the text.

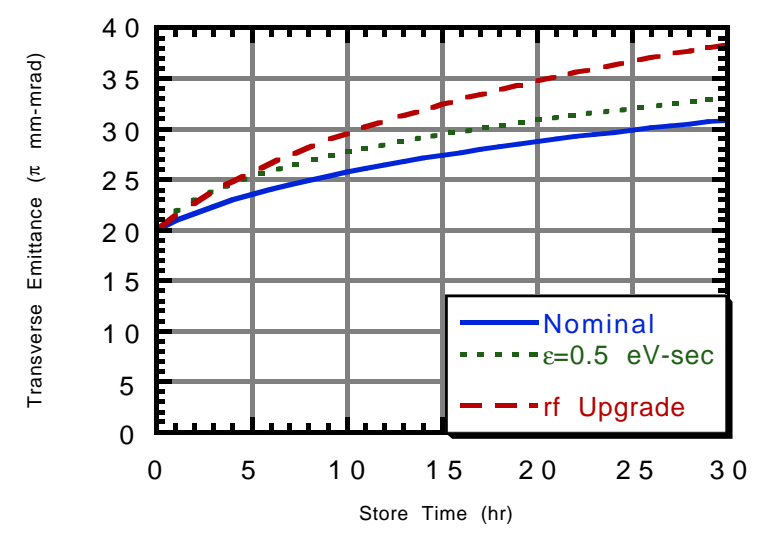

Figure 6. The time dependence of the transverse emittance of the stores described in the text.

\section{Luminosity leveling}

The peak luminosity of $10^{33} \mathrm{~cm}^{-2} \mathrm{sec}^{-1}$ with 100 bunches per beam results in about 10 interactions per bunch crossing. It is difficult (and expensive) to build high performance detectors to operate in this environment. One possible operational scenario involves a technique known as "luminosity leveling". The idea is that the luminosity is held at a maximum valuesay $0.5 \times 10^{33} \mathrm{~cm}^{-2} \mathrm{sec}^{-1}$-during the initial part of the store and kept constant for as long as possible. One straight-forward method of accomplishing this goal is to dynamically adjust the value of $\beta^{*}$ as the store progresses. The luminosity is kept constant until the minimum value of $\beta^{*}$ is achieved. A simulation of luminosity leveling is shown in Figure 7 for the nominal parameters in Table I. The loss of luminosity from intrabeam scattering and residual gas effects is the same for both stores, but the luminosity limited store retains more 


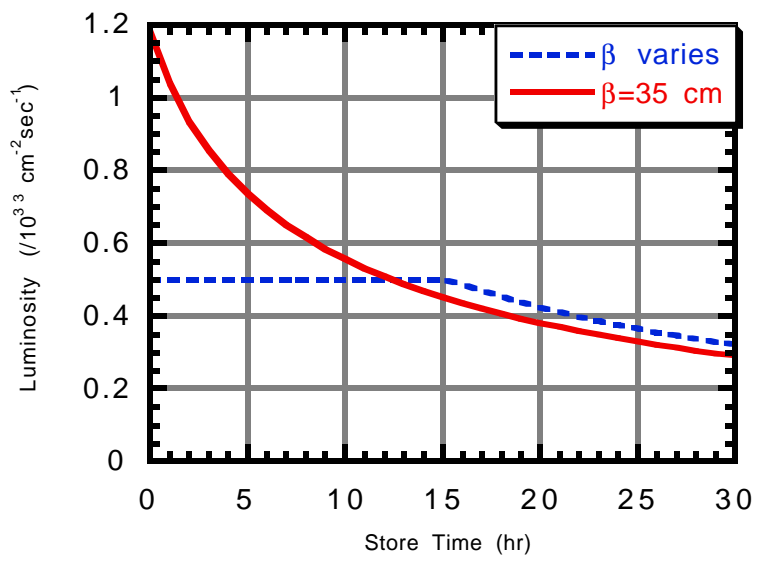

Figure 7. A store with the nominal parameters and $N_{\bar{p}}=27 \times 10^{10}$ is compared to an otherwise identical store where $\beta^{*}$ is varied to maintain a constant luminosity of $0.5 \times 10^{33} \mathrm{~cm}^{-2} \mathrm{sec}^{-1}$ for as long as possible keeping $\beta^{*}>35 \mathrm{~cm}$.

antiprotons (fewer collisions) and has a higher luminosity at long times in the store.

The limited store yields $23.4 \mathrm{pb}^{-1}$ in 14.0 hours (a 13 hour store plus one hour for shot setup) for an average luminosity of $1.67 \mathrm{pb}^{-1} / \mathrm{hr}$ compared to $36.2 \mathrm{pb}^{-1}$ in $15.6 \mathrm{hr}$ and an average luminosity of $2.32 \mathrm{pb}^{-1} / \mathrm{hr}$ for the unlimited store. The average luminosity obtained with a luminosity leveled store is less sensitive to the initial antiproton bunch intensity than a unleveled store, but the highest integrated luminosity is obtained in either case with the highest possible initial antiproton intensity. The loss of luminosity from leveling depends on the store parameters: the importance of the antiproton intensity to the lifetime, the amount of luminosity reduction desired, and the length of the store.

\section{B. The beam-beam interaction}

A crucial issue for TeV33 is the increased number of bunches in the Tevatron. As the number of bunches increases so do the number of crossings with long-range beam-beam interactions, and it becomes more difficult to avoid adverse effects caused by these interactions. Although the number of interaction points is expected to remain at two or possibly be reduced to one, the number of long-range collisions will increase to about 200 for the TeV33 configurations now envisioned.

The many "near-miss" collisions produce normal and skew quadrupole components which change the tunes and coupling, and dipole kick components which modify the separation between beams and change the dispersion. Higher order effects, such as chromatic effects, are also present, but are not evaluated in this work. All of these effects differ from bunch to bunch because the bunches are irregularly spaced.

In this work, we examine the effects of the beam-beam interaction in an approximate way to take a first look at the scope of the problem and to make preliminary judgments about potential solutions. Attention is focused on methods of reducing the tune space occupied by the antiproton beam.

It should be emphasized that this analysis is not exhaustive, and contains approximations that should be removed before final design decisions are made. Non-linear effects of the beambeam interaction were not studied beyond the calculation of the tune spread. For example, synchro-betatron resonances [4] are excited when a non-zero crossing angle is introduced. The dependence of the antiproton tune shifts on proton beam emittance was not studied. These effects may be important considerations in a more detailed study.

\section{Bunch Loading Scenarios}

The bunches in TeV33 will probably be spaced at $7 \mathrm{rf}$ bucket $(39.5 \mathrm{~m})$ intervals. The trigger electronics for the two major detectors (CDF and D0) are being upgraded for a $7 \mathrm{rf}$ bucket spacing. Smaller spacing between bunches or even unbunched beams have been discussed but are not considered here. A gap in the beam to accommodate injection and extraction (the beam abort) is required. At the termination of a normal store, only the protons are removed, and they will probably be removed without the use of the abort kickers. However, the abort kickers will still be used in abnormal situations to remove the beams quickly without quenching the superconducting magnets.

The existing beam abort kickers require a minimum gap of $2.6 \mu \mathrm{sec}$ (139 rf buckets) to rise to a field which is adequate to steer the beam onto the abort dump. The major experimental areas are located at B0 and D0: 1/3 of the circumference of the ring apart. Loading the ring in a three-fold symmetric fashion provides an identical pattern of interactions at the two interaction regions. Non-symmetric patterns are possible and can have some advantages; a non-symmetric pattern would almost certainly be used if there were only one detector. However, in this paper we will consider a specific three-fold symmetric distribution. The bunch distribution is further constrained by the need to allow for the injection kicker rise time.

The bunch loading scheme used in the present analysis, which satisfies the above constraints, calls for three groups of bunches, each $371 \mathrm{rf}$ buckets long to fill the 1113 Tevatron rf buckets. A group consists of 3 batches of 10 bunches spaced at $7 \mathrm{rf}$ bucket intervals. The batches are separated by $20 \mathrm{rf}$ buckets for the injection kicker and the last batch is followed by the abort gap of 139 empty buckets.

\section{IR Optics \& Crossing Angles}

The present Tevatron IR design achieves zero dispersion $\left(\eta^{*}=0\right)$, but the slope $\eta^{* \prime} \neq 0$. The non-zero slope results in the dispersion reaching its maximum value within the low-beta triplet quadrupoles. In this study we use an alternative IR design $[5,6]$, which uses the existing magnet configuration and yields $\eta^{*}=\eta^{* \prime}=0$. The IR lattice functions for this dispersionfree solution with $\beta^{*}=35 \mathrm{~cm}$ are shown in Figure 8 .

The first parasitic crossing for TeV33 occurs just $19.8 \mathrm{~m}$ from the interaction point (IP), placing it within the interaction region (IR) triplet quadrupoles and before the first 


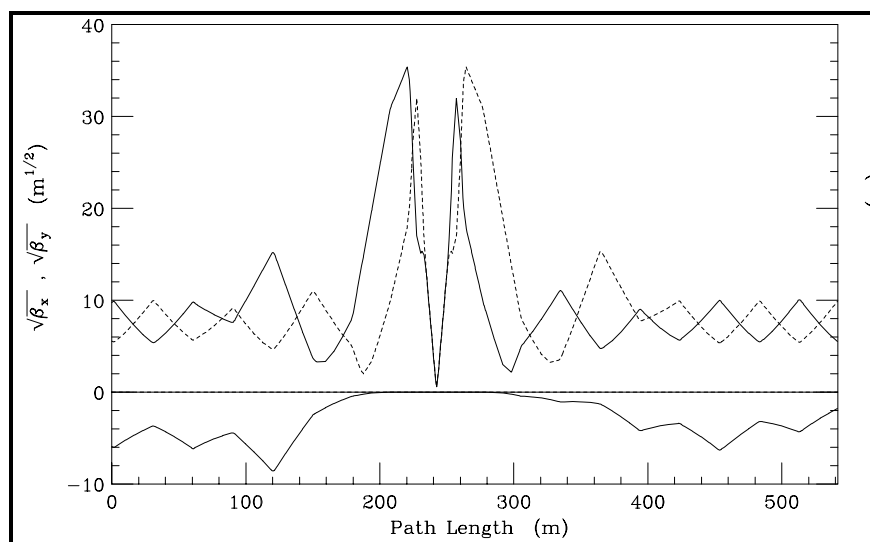

Figure 8. Dispersion-free IR lattice functions for $\beta^{*}=35 \mathrm{~cm}$.

set of separators. It currently does not appear possible to avoid collisions at these points without introducing a crossing angle at the IP. A straightforward calculation [5] shows that additional separators inboard of the IR triplet quadrupoles would have little effect on beam separation at the first crossing. Essentially all the betatron phase advance occurs in the immediate vicinity of the IP, and is practically zero across the IR triplet quadrupoles. For realistic gradients and lengths, electrostatic separators are incapable of producing the 3-5 $\sigma$ beam separation desired, with $\theta=0$ at the IP. Without a crossing angle the additional crossing points would more than triple the beam-beam tune shift arising from the interaction point.

Ultimately, the size of the crossing angle will be determined by operational experience. A priori, this choice is not obvious. The crossing angle represents a compromise between avoiding excessive beam-beam effects from the parasitic crossings nearest the interaction point and the reduction in luminosity resulting from a crossing angle. The reduction in luminosity and the beam-beam tune shift from the collision at the IP are shown versus crossing angle in Figure 9.

The half-crossing angle $\theta$ that produces $n \sigma$ beam separation at the first parasitic crossing is approximately:

$$
\theta \approx \frac{n}{2} \cdot \sqrt{\frac{\varepsilon}{6 \pi \beta^{*} \beta_{r} \gamma_{r}}}
$$

where $\beta_{r}=v / c \approx 1$. For $3 \sigma$ and $5 \sigma$ separations $\theta$ becomes approximately 142 and $236 \mu \mathrm{rad}$, respectively. We have chosen to divide the crossing angle equally between the horizontal and vertical planes at each IP $\left(\theta_{x}=\theta_{y}=100\right.$ and $170 \mu \mathrm{rad}$, respectively). This choice is certainly not unique, and extensive additional study is required to determine the optimum configuration.

\section{Tune Shift Calculations}

The beam-beam dipole kicks, tune shifts, and coupling shift are determined for each bunch from the widely used expression for the beam-beam potential [7]



Figure 9. The dependence of the luminosity and the beambeam tune shift for zero amplitude particles from the interaction point crossing is shown. The crossing half-angles in the $\mathrm{x}$ and $\mathrm{y}$ planes are equal $\left(\theta_{x}=\theta_{y}\right), \beta^{*}=35 \mathrm{~cm}, \sigma_{s}=35 \mathrm{~cm}$, and $\varepsilon=20 \pi \mathrm{mm}-\mathrm{mrad}$.

$$
U(x, y)=\frac{N r_{p}}{\gamma_{r}} \int_{0}^{\infty} d q \frac{1-\exp \left[\frac{-x^{2}}{2 \sigma_{x}^{2}(1+q R)}+\frac{-y^{2}}{2 \sigma_{y}^{2}(1+q / R)}\right]}{\sqrt{(1+q R)(1+q / R)}},
$$

where $r_{p}$ is the classical proton radius $\left(1.535 \times 10^{-18} \mathrm{~m}\right), N$ is the number of particles per bunch, $\gamma_{r}$ is the relativistic energy factor, and $R$ is the ratio of vertical to horizontal beam size $\left(\sigma_{y} / \sigma_{x}\right)$. The dipole kick in the $x$ plane is

$$
\Delta x^{\prime}=-\frac{\partial U}{\partial x}
$$

with a similar formula for the $y$ plane.

We have calculated the beam-beam effects in this section using the parameters listed in Table I with $N_{\bar{p}}=6 \times 10^{10}$. The longitudinal emittance was changed to $2 \mathrm{eV}$-sec because smaller emittances are expected from the Main Injector, and $B=90$ from the bunch loading scheme described in sub-section 1 above.

We use an iterative approach to calculate self-consistent bunch-by-bunch closed orbits. The beam-beam dipole kicks have a significant effect on the separation between the beams at the IP's. Uncorrected, they would cause separations at the IP of about a beam sigma and would change 2 of the 4 crossing angles by about $200 \mu \mathrm{rad}$. We adjust the separator settings to correct the average separations and angles. After we've corrected the average separations and angles, there are still bunch-to-bunch variations. These are shown for the separations at B0 in Figure 10. The non-zero separations reduce the luminosity of that bunch. However, the effect is fairly small: there is a $6 \%$ reduction in luminosity for a 20 $\mu \mathrm{m}$ offset. The effects on the angular separation and the changes at D0 are similar in magnitude but differ in detail. 


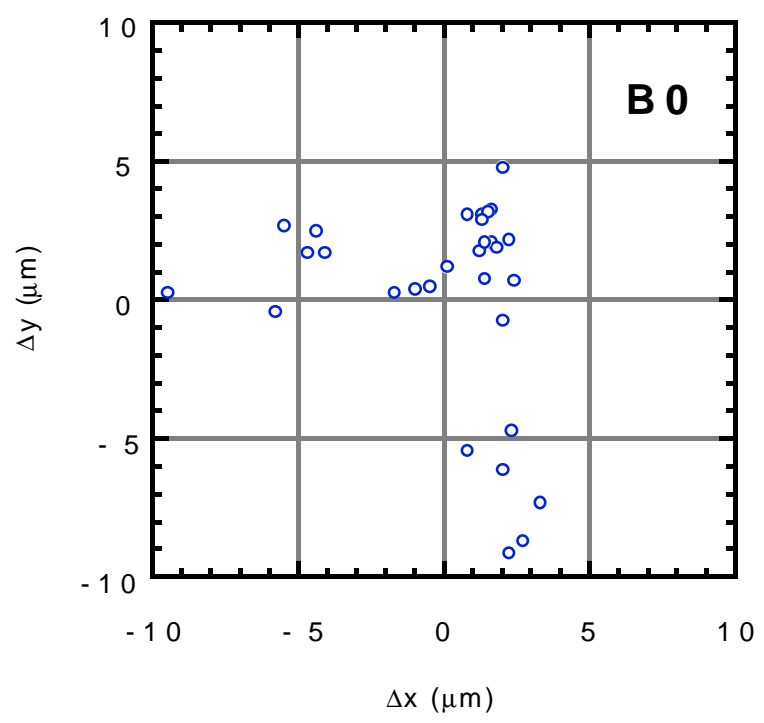

Figure 10. The transverse separation at the B0 interaction point between each antiproton bunch and the average position of the proton beam. The rms beam size of a $20 \pi \mathrm{mm}-\mathrm{mrad}$ beam is $33 \mu \mathrm{m}$ at $\mathrm{B} 0$.

We define the diagonal sigma separation of the beams $(d)$ from $d^{2}=\Delta x^{2} / \sigma_{x}^{2}+\Delta y^{2} / \sigma_{y}^{2}$, where $\Delta x(\Delta y)$ is the distance between the beams and $\sigma_{x}\left(\sigma_{y}\right)$ is the rms beam size. It is a rough, but traditional, figure of merit used to gauge the strength of the long-range beam-beam interactions. The diagonal sigma separation is shown in Figure 11. The points with 0 separation are the collision points at B0 and D0, but the small sigma separation near the collision points $(2.3 \sigma$ separation at rf bucket 735) is a concern.

The tunes of a small amplitude particle can be written as

$$
\begin{aligned}
v_{ \pm} & =\left[\left(v_{x}+\Delta v_{x}\right)+\left(v_{y}+\Delta v_{y}\right)\right] / 2 \\
& \pm \sqrt{\left[\left(v_{x}+\Delta v_{x}\right)-\left(v_{y}+\Delta v_{y}\right)\right]^{2} / 4+|\kappa+\Delta \kappa|^{2}}
\end{aligned}
$$

where $v_{x}$ and $v_{y}$ are the unperturbed horizontal and vertical tunes, $\kappa$ is a complex number describing the coupling and the $\Delta$ 's represent the changes to these quantities that arise from the beam-beam force. The horizontal tune shift is found from the approximation:

$$
\Delta v_{x}=-\frac{\beta_{x}}{4 \pi} \frac{\partial^{2} U}{\partial x^{2}},
$$

and similarly for the vertical tune. Because the beams are separated both horizontally and vertically at a typical crossing point, the beam-beam interaction generates transverse coupling shifts. The tunes are sensitive to these shifts at our normal operating point $v_{x}-v_{y} \approx 0.01$. The coupling shift is calculated from

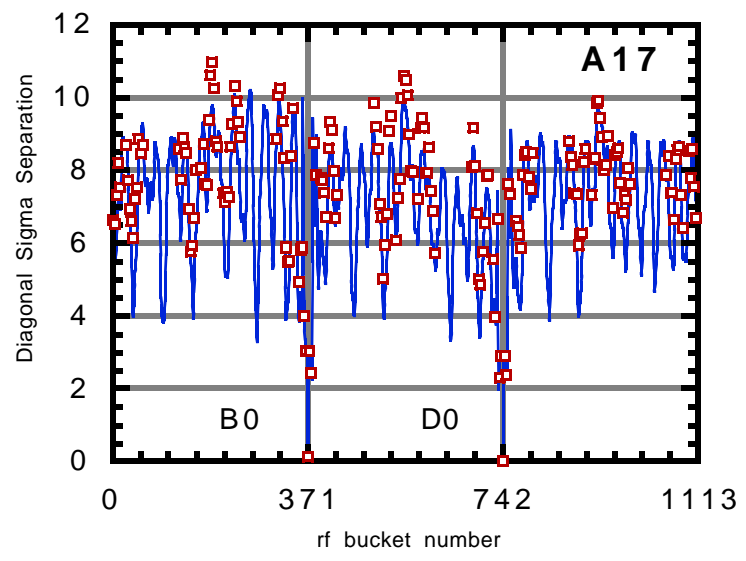

Figure 11. Diagonal sigma separation in the Tevatron as a function of azimuthal position. The line shows the diagonal sigma separation of the nominal orbit at all 2226 potential crossing points. The markers show the diagonal sigma separation for a particular bunch (the 17th bunch location in the group) at its 180 crossing points after including the beambeam dipole kicks and readjusting the separator voltages to bring the beams into collisions.

$$
\Delta \kappa=\frac{\sqrt{\beta_{x} \beta_{y}} e^{i\left(\psi_{x}-\psi_{y}\right)}}{4 \pi} \frac{\partial^{2} U}{\partial x \partial y} .
$$

Each of the quantities in Eqns. 8 and 9 is computed for each bunch crossing and then summed over all 180 bunch crossings (including the two interaction points). The dependence of the tune and coupling shifts on the crossing angle at the IP is significant and has been included in the calculations. The shifts in tune and coupling are shown in Figure 12 and Figure 13.

At the beam crossings nearest the interaction points the separation between the beams is small, and the beam-beam effects are strong. Because we have chosen equal magnitude horizontal and vertical crossing angles, these crossings contribute large coupling changes (about 0.004) but only small tune changes. We have chosen the signs of the horizontal and vertical crossing angles at B0 and D0 so that the transverse coupling changes from B0 largely cancel those from D0. This "trick" greatly helps to keep these crossings manageable, but depends on having an even number of IP's.

If we know the dependence of the tune on oscillation amplitude, we can determine the tune spread of the beam. The tune shift is proportional to the derivative with respect to action of the beam potential (Eqn. 5) averaged over the particle motion. The shift is easily computed with the ansatz that the average may be found using the unperturbed motion (instead of the actual motion including beam-beam effects). Since the usual Tevatron operating point is near the coupling resonance, this approximation may not be very accurate. We have not 


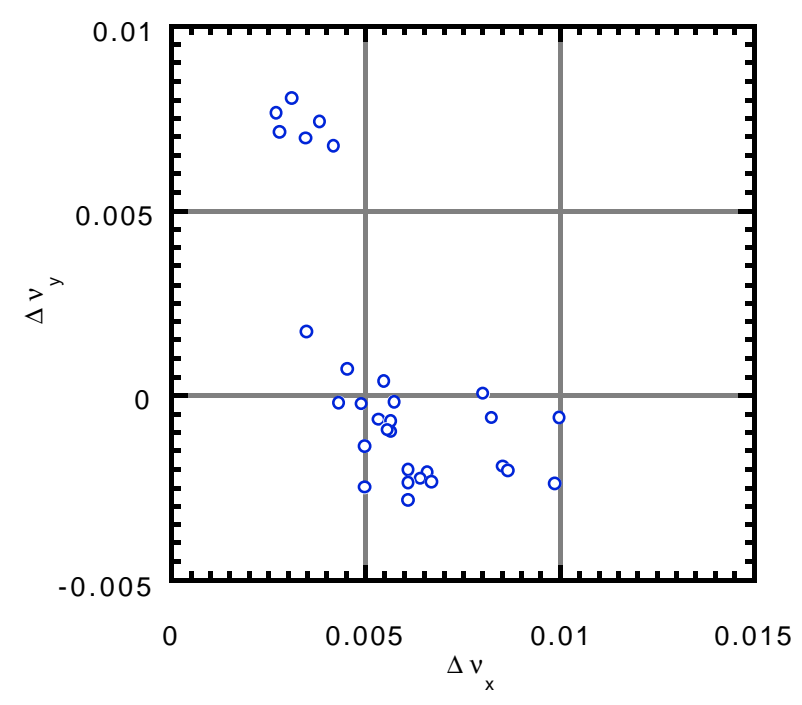

Figure 12. The horizontal and vertical tune shifts for a zero amplitude antiproton in each of the 30 bunches in a group. The tune shift includes the 2 interaction points and the 178 long-range interactions.

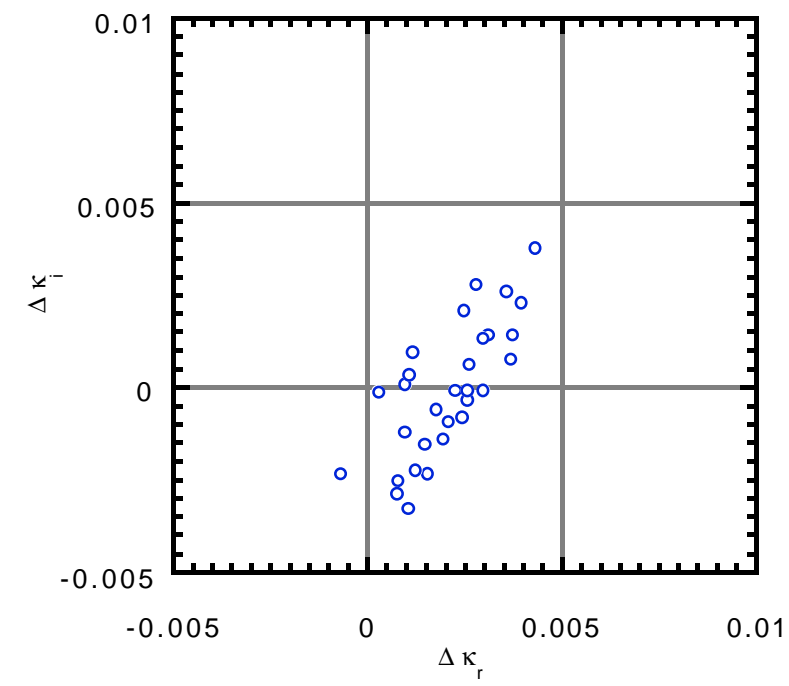

Figure 13. The complex coupling shifts for a zero amplitude antiproton in each of the 30 bunches in a group. The coupling shift includes the 2 interaction points and the 178 long-range interactions.

considered the behavior of off-momentum particles in these calculations.

Most of the tune spread appears to arise from the long-range beam-beam interactions. As an example, Figure 14 shows the contribution to the beam-beam tune spread from the collisions at the two IP's for antiproton bunch number 17 in the group of 30 bunches. A grid of lines indicates the tunes of particles with different amplitudes. Each line represents the tune of particles with constant amplitude in $x$ or $y$ and varying

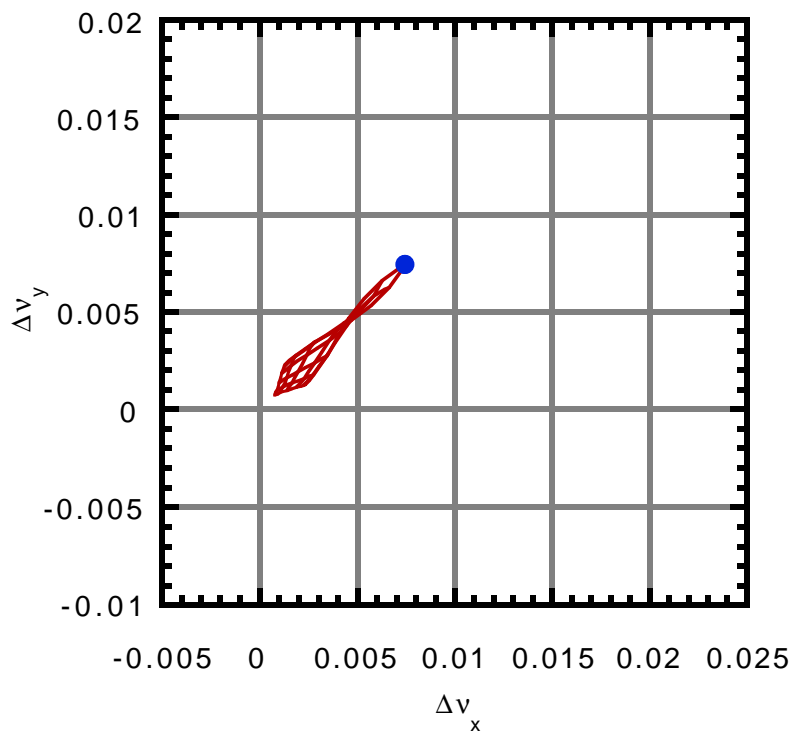

Figure 14. Tune grid for bunch 17 showing the contribution of the collisions at the 2 interaction points. The grid lines are spaced by $1 \sigma$ in the oscillation amplitude. The tune shift of the zero amplitude particle is shown by the large dot.

amplitude in the other plane. The grid is drawn in steps of $1 \sigma$, where $\sigma$ is the rms beam size in the respective plane of motion. Figure 15 is the corresponding plot for the long-range interactions. The tune shift arising from the beam-beam interaction at the IP decreases with increasing amplitude while the long-range interaction tune shift does the opposite.

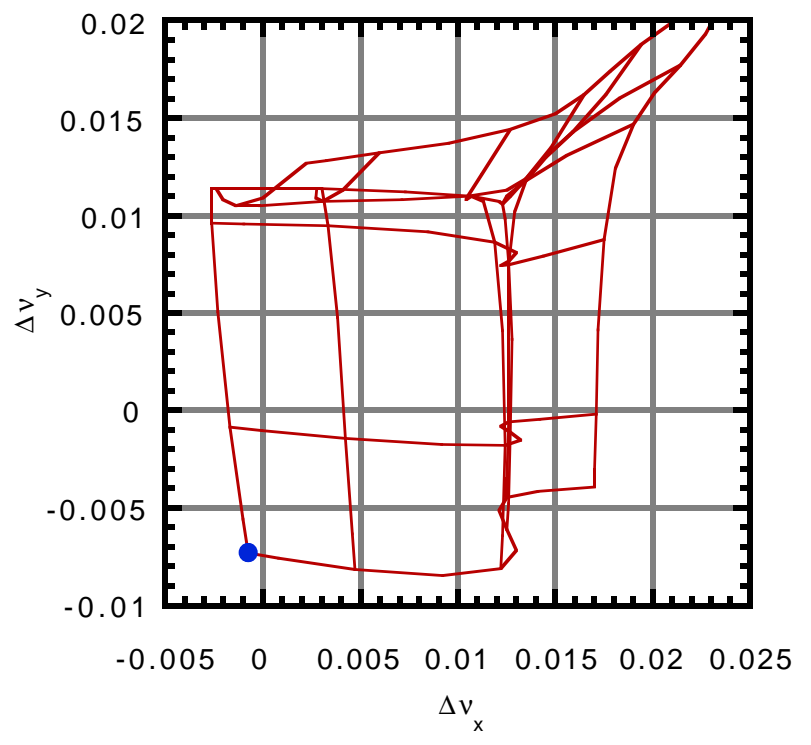

Figure 15. Tune grid for bunch 17 showing the contribution of the 178 long range interactions only. The grid lines are spaced by $1 \sigma$ in the oscillation amplitude. The tune shift of the zero amplitude particle is shown by the large dot. 


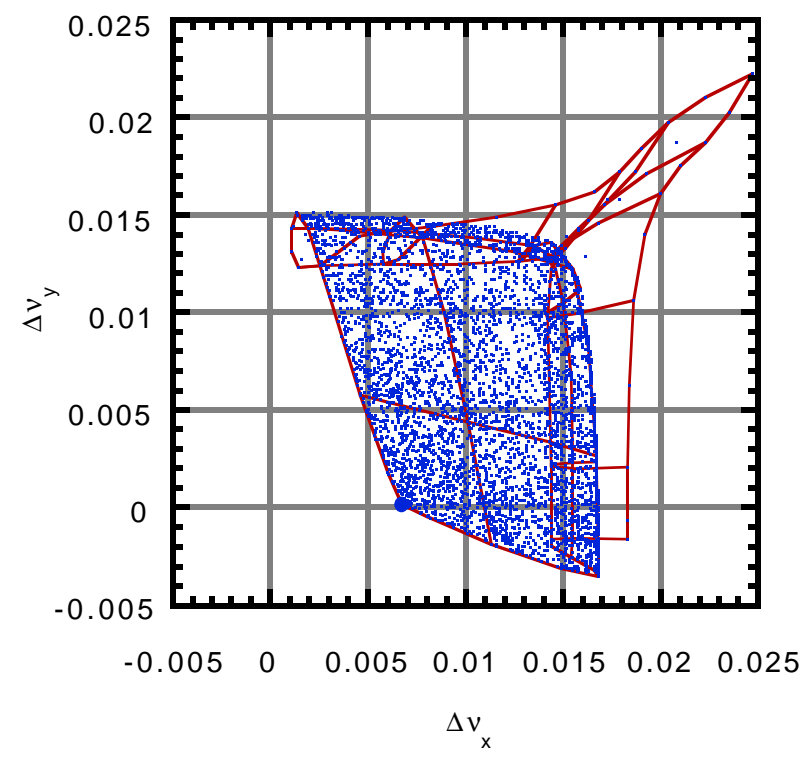

Figure 16. Tune grid and distribution of particle tunes for all 180 crossing points of antiproton bunch 17 . The tune distribution is calculated for 5000 particles whose amplitudes were randomly generated from a gaussian beam distribution. The tune shift of the zero amplitude particle is shown by the large dot.

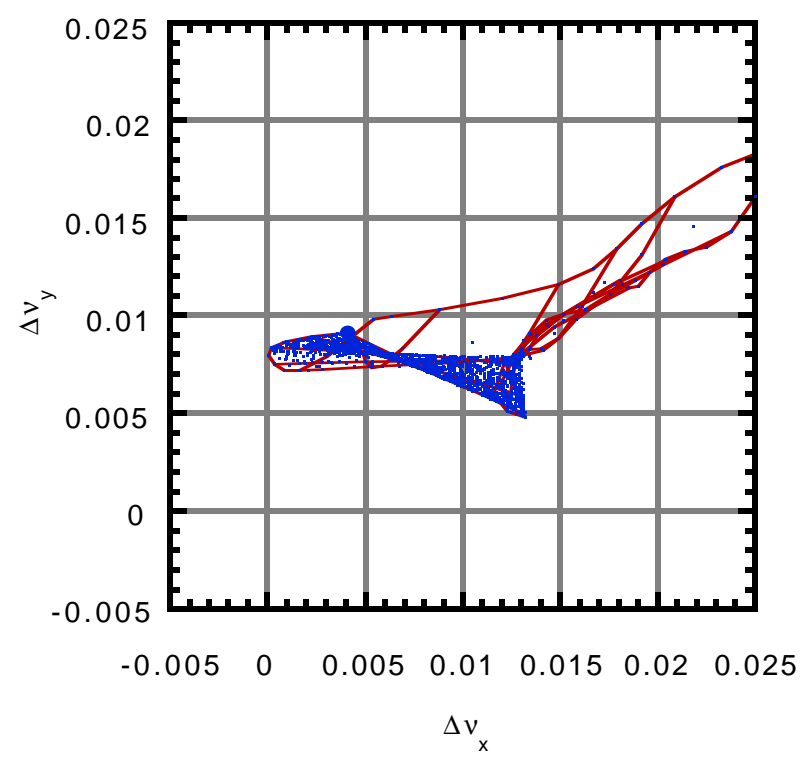

Figure 17. Tune grid and distribution of particle tunes for all 180 crossing points of the first antiproton bunch. The tune distribution is calculated for 2000 particles whose amplitudes were randomly generated from a gaussian beam distribution. The tune shift of the zero amplitude particle is shown by the large dot.
In order to get a better picture of the distribution of tunes, the tune grid plot can be overlaid with points representing a gaussian beam distribution. These plots are shown in Figure 16 (bunch 17), Figure 17 (the first bunch), and Figure 18, (the last bunch). Figure 19 shows the combined tune distribution for all the antiproton bunches.
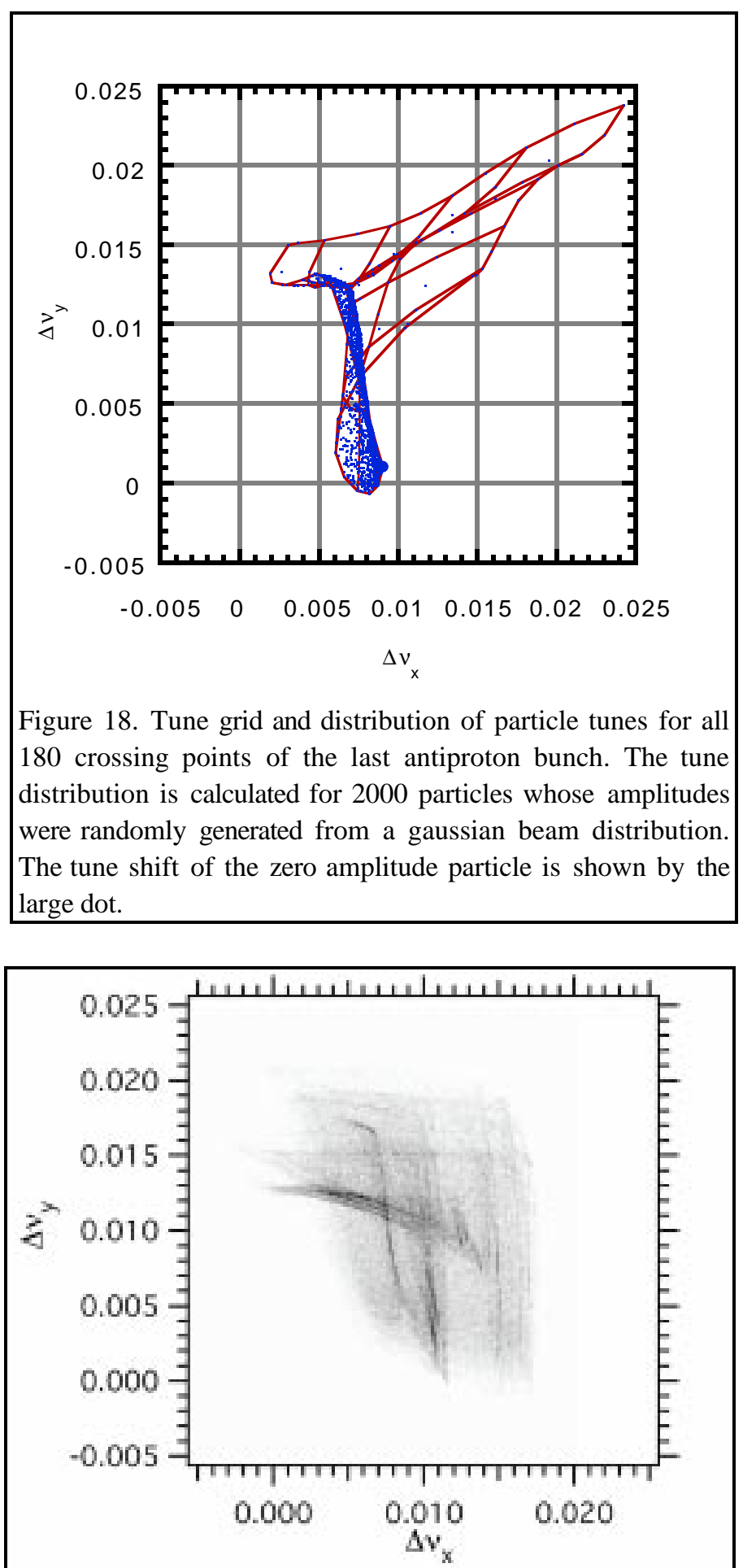

Figure 19. An intensity plot of the distribution of tunes for all 30 antiproton bunches. 
The tune distribution appears to be compatible with a working tune space of 0.025 , but we are not confident that the bunch loading and beam separation configuration described will provide adequate performance for colliding beam physics experiments. Compared to a regular Cartesian grid, the tune grids are highly distorted and folded. The highly non-linear beam-beam effect may drive unusually high order resonances and reduce the tune working space. At the folds, the tune is independent (to first order) of the amplitude. These portions of the beam may be more sensitive to resonant and coherent effects than is normally the case.

We believe that we have made significant progress in understanding the complicated beam-beam interaction that will be present during TeV33 operations. However, we still have most of our work ahead of us.

1. The tune spreads calculated are not necessarily the smallest ones that could be obtained. We need to explore different configurations of separator setting and possible modifications to magnets and separators to obtain better beam separation.

2. The effects of the beam momentum spread and dispersion need to be included in the calculations.

3. The tune spread calculations need to take into account coupling.

4. Approximate calculations need to be supplemented with tracking simulations.

5. The effect of synchro-betatron resonances need to be considered in more detail.

6. The effects of "near-miss" collisions (less than, say, $3 \sigma$ beam separation) need to be considered in more detail.

\section{Slip Stacking}

Slip stacking is a method that has been proposed to increase the proton intensity at the antiproton target. In this method two Booster batches are injected at different azimuths and momenta and captured by two independent rf systems. Since the momenta and therefore the rf frequencies are different, the batches "slip" past each other. As the batches approach the same azimuth, the momentum difference is decreased, and the corresponding bunches of the two batches are captured by the large $\mathrm{rf}$ buckets of a single rf system. The process does not require improved Booster performance but requires the large momentum aperture that will be available in the Main Injector.

The single particle dynamics of slip stacking have been studied in some detail, and the results are reported in a separate paper in these proceedings [8]. The result of the simulation suggests that it will be possible to obtain 1.95 times the single batch proton intensity in $230 \%$ of the longitudinal emittance of a single batch (assumed to be $0.15 \mathrm{eV}$-sec per bunch). The rf voltages required are low, so it will be necessary to have the rf cavities well compensated for beam loading. Other collective effects still need to be evaluated.

\section{Antiproton Production}

We plan to dramatically increase antiproton production for TeV33, with stack rates up to $100 \times 10^{10}$ antiprotons/hr. This

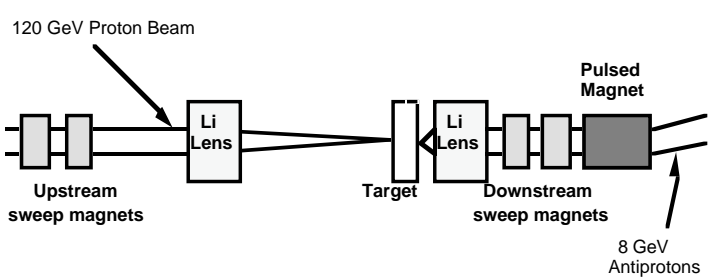

Figure 20. Components in the target vault of the upgraded target station. Not shown are the target secondary emission monitor (SEM) and the beam dump.

rate may be achieved with a combination of increased proton beam at the antiproton production target and increased antiproton acceptance. As the intensity on target increases, measures to protect the target station components from damage, particularly radiation damage, become increasingly important.

Figure 20 shows a layout of the target station after the completion of the Run II upgrades. The AP1 beam line will transport and focus the $120-\mathrm{GeV}$ protons from the Main Injector onto the target. Antiprotons created in the target are collected by a lithium lens, and deflected by the pulsed magnet into the AP2 beam line for injection into the Debuncher at 8.9 $\mathrm{GeV} / \mathrm{c}$. The upstream sweep magnets will be installed at the end of the AP1 beam line near the focal point of the proton lens. The downstream magnets will be located at two currently unoccupied modules between the collection lens and the pulsed magnet, near the focal point of the collection lens.

\section{Antiproton Acceptance}

Two steps are planned to improve the antiproton yield for TeV33. The yield is defined as the number of antiprotons collected per targeted proton. The first improvement concerns the collection lens, which focuses the antiprotons produced at the target. The current-carrying portion has a length of $15 \mathrm{~cm}$ and a radius of $10 \mathrm{~mm}$. Fermilab lithium lenses of recent design have survived over 7 million pulses with an edge field of about 7.5 T. Increasing the strength of the lens increases the yield. But even a small increase in repetitive stress in the water-cooled titanium cylinder that encases the lithium results in a significant reduction in the fatigue life of the metal. A number of recent improvements in the design of the lens [9] are expected to allow operation up to about $9 \mathrm{~T}$. The most important improvements are changes in the mechanical construction and filling procedure and the reduction of radiation-induced swelling by using ${ }^{7} \mathrm{Li}$ instead of the naturally occurring abundances of the isotopes. The combined yield improvement due to collection lens improvements is expected to be $15 \%$. 
The second improvement [10] is to increase the transverse acceptance of the AP2 beam line and the Debuncher Ring to $32 \pi$ from $17 \pi \mathrm{mm}-\mathrm{mrad}$ (95\% emittances, unnormalized). ${ }^{*}$ The transverse aperture of the Debuncher is now about $25 \pi$, limited by the stochastic cooling tanks. We plan to upgrade the cooling tanks with plunging pickups and kickers to increase the nominal aperture of the tanks to $40 \pi \mathrm{mm}$-mrad. It is likely that other improvements, such as realignment of the Debuncher magnets, redesign of the injection/extraction kickers, and additional trim magnets, will be required to achieve the goal of $32 \pi \mathrm{mm}$-mrad aperture. Currently, the limiting factor in the transverse acceptance is the AP2 line. It is believed that realignment of the beam line magnets will remove this acceptance limitation.

The increase in acceptance was calculated to increase the collection efficiency by a factor of 1.9. Combining the increase in acceptance with the improvements in the lithium lens, we expect an increase of a factor of 2.2, based on the Monte-Carlo simulation MCLENS [11].

The following potential upgrades may provide additional yield increases, but have not been factored into the yield predictions because of uncertainty in their development.

1. The optics match between the target station and the AP2 beam line is probably not optimum at present. Correction of the beam line optics by providing shunts for some of the quadrupoles will offer additional flexibility in matching the distribution of particles from the target station $\left(\beta_{x}=\beta_{y}=4 \mathrm{~m}\right)$. Improving the match is calculated to increase the collection efficiency by a factor of about 1.15 (see Figure 21).

2. Fermilab has recently begun to consider the development of a liquid lithium lens [12], which has the potential to operate at a much higher edge field than a conventional lens. A series of calculations is shown in Figure 21, showing the effect of various options on operation of the lens. The existing lens (15-cm length, 10-mm radius) and AP2 optics are shown as the large black circle. The effect of matching AP2 optics to the existing lens geometry is to increase the yield by roughly $15 \%$ and lower the optimal lens field. Better performance is expected with a high-field liquid lithium lens as the lens is shortened (10-cm length, $10-\mathrm{mm}$ radius). A miniaturized (6-cm length, 6-mm radius) lens obtains even higher yields. The optimum edge field is very high, and this lens may not be achievable in practice. The matching beta functions for AP2 in this case are $1.5 \mathrm{~m}$, and the potential additional yield improvement might be $15 \%$.

3. It may be possible to increase the momentum acceptance of the Debuncher from the current 4\%. Run II plans call for ramping the Debuncher lattice to increase

\footnotetext{
*The emittances quoted in section D are the phase space areas containing $95 \%$ of the beam and are not normalized by the beam energy.
}

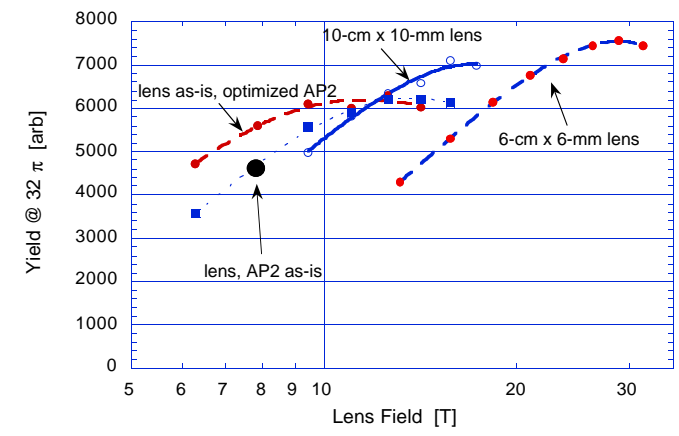

Figure 21. Yield curves for some collection lens scenarios, assuming fixed $32 \pi$-mm-mrad AP2/Debuncher acceptance.

$\eta=1 / \gamma_{t}^{2}-1 / \gamma^{2}$ from .006 during bunch rotation to .009 during the cooling portion of the cycle.

4. Finally, some of the schemes proposed to increase Main Injector beam intensity on target take advantage of its large transverse aperture. If the emittance of the proton beam is significantly increased beyond $(20 \pi \mathrm{mm}-\mathrm{mrad}$ normalized emittance), it will be necessary to use a proton lens to focus the beam to a small spot size on the target. Beam loss in the proton lens is expected to be $7.5 \%$.

\section{Target Damage and Beam Sweeping}

In addition to improving collection efficiency, it will be necessary to target a greater number of $120-\mathrm{GeV}$ protons. The Main Injector is projected to initially target $5 \times 10^{12}$ protons in a 1.6- $\mu$ sec pulse. The upgrade schemes will result in increases in proton intensity of factors of 2-4 (slip stacking, betatron stacking, etc.), or a factor of 6 in a longer $(10 \mu \mathrm{sec})$ pulse. The concerns arising from increased beam intensity fall into three categories: (1) instantaneous damage to the target, (2) radiation damage to target and target-station components, and (3) radiation shielding considerations.

Reducing the size of the proton beam spot at the target increases the efficiency of collecting antiprotons from the target. However, it also increases the peak energy deposition. To reduce the density of energy deposition to current levels, we plan [13] to rapidly sweep the beam on the target.

The estimated peak energy density deposited per pulse is now $800 \mathrm{~J} / \mathrm{g}$. This density is above the melting point of copper (about $600 \mathrm{~J} / \mathrm{g}$ ) and close to the melting point of nickel (about $1000 \mathrm{~J} / \mathrm{g}$ ). In order to maintain peak energy deposition below present levels, the TeV33 spot size will have to be either increased substantially or swept on the target.

In the absence of beam sweeping, the yield degrades as the intensity increases because the beam spot size must be increased. Sweeping allows a reduction in spot size and the restoration of yield. As the beam intensity increases, sweeping becomes increasingly important, and larger radii are required to completely restore the yield to its low intensity level. Figure 22 shows estimates of yield for several sweep 


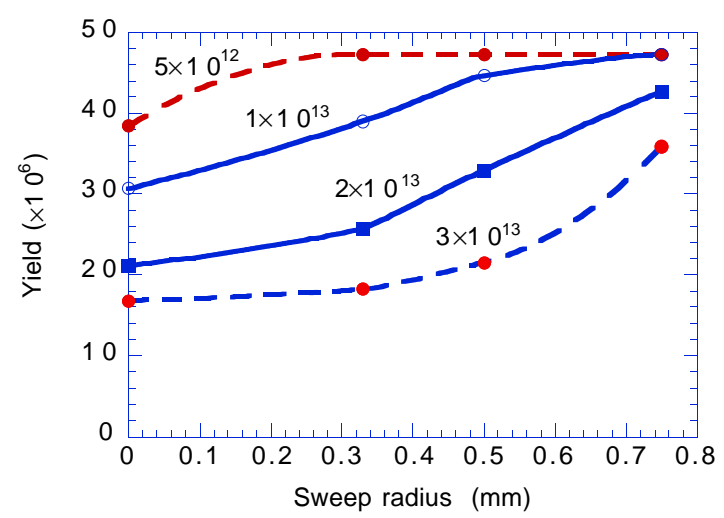

Figure 22. Yield into a $32 \pi \mathrm{mm}$-mrad acceptance vs sweep radius. The peak target energy density is limited to $800 \mathrm{~J} / \mathrm{g}$, the lens edge field is $9.6 \mathrm{~T}$, the minimum proton beam size at the target is $0.15 \mathrm{~mm}(\mathrm{rms})$, and the AP2 line is assumed to be matched to the lithium lens.

radii and beam intensities while varying the proton beam size to maintain a fixed energy deposition of $800 \mathrm{~J} / \mathrm{g}$.

The beam sweeping scheme currently under development traces a $0.33-\mathrm{mm}$ radius circular pattern on the target over the $1.6 \mu \mathrm{sec}$ proton beam pulse. A pair of orthogonal kickers are excited in quadrature by $6-\mathrm{kA}, 6-\mathrm{kV}$ sinusoidal wave-forms. The high voltage required is a matter of some concern because ionization of the air by the particle shower downstream of the target increases the conductivity of the air between the conductors. An alternative rotating magnetic field design has been proposed to combine the functions of the two kickers into a single magnet [14]. Among the advantages of this design is that a $50 \%$ larger sweep radius can be achieved for a given excitation voltage. It may therefore be possible to sweep a radius of $0.5 \mathrm{~mm}$, which is important for operation at the higher proton intensities. A larger sweep radius (say 0.75 $\mathrm{mm})$ may be possible for a slower $(10 \mu \mathrm{sec})$ beam pulse because the operating voltage is dominated by the inductance of the magnet.

Figure 23 shows the predicted number of antiprotons collected per hour as a function of protons per pulse, assuming the upgrades described above, and a 1.5-sec cycle time. It shows that the rate of production of antiprotons could be in the desired range of $1 \times 10^{12}$ per hour for a proton intensity on target somewhat above $1 \times 10^{13}$ per pulse, assuming a sweep radius of at least $0.5 \mathrm{~mm}$.

\section{Radiation Damage Issues}

The matters of greatest concern for radiation damage to target station components are:

1. Rotation of the target on a pulse-to-pulse basis is necessary to expose fresh target material to the proton beam. Past episodes of serious damage have occurred when the target rotation mechanism failed because of



Figure 23. Antiprotons collected in the Debuncher for several sweep radii. Assumptions: $800 \mathrm{~J} / \mathrm{g}, 32 \pi$ acceptance, lens edge field of $9.6 \mathrm{~T}, 0.15-\mathrm{mm}$ spot size, optimized AP2, $1.5 \mathrm{sec}$ cycle time.

swelling of bearings. Operation with graphite bushings to replace the bearings may help alleviate this problem.

2. In the lithium lens, gaseous products from the ${ }^{6} \mathrm{Li}(\mathrm{n}, \alpha){ }^{3} \mathrm{H}$ reaction are expected to build up over time. Swelling of the lens is expected, driven by the pressure of the trapped hydrogen gas. The increased internal pressure limits the lifetime of the lens by increasing operating stress. To avoid this potential problem, we now fill lenses with $99 \%$ pure ${ }^{7} \mathrm{Li}$ instead of the naturally occurring isotopic abundance.

3. Insulating materials, such as kapton ${ }^{\circledR}$ and torlon ${ }^{\circledR}$, used in the collection lens, sweep magnet, pulsed magnet and other electrical devices will be irradiated well past their level of susceptibility to radiation damage. Operation at increasing irradiation levels may shorten the lifetimes of these devices dramatically. Some relief may be obtained by using ceramic insulation, where possible.

\section{Radiation Safety and Environmental Issues}

Targeting high intensity proton beams requires a careful examination of safety and environmental issues. Some of the more important issues are listed below.

1. Radiation shielding in the target hall is designed for intensities of $5 \times 10^{12}$ at a repetition rate of $1.5 \mathrm{sec}$. Increased intensity will require an increase in the amount of shielding in and around the target vault, stairwells, penetrations, and beam lines.

2. Based on a concentration model [15], intensity limits due to groundwater activation do not appear to be a problem. The model indicates a limit of $10^{22}$ protons per year on target, well above the most extreme projection for proton intensity $\left(5 \times 10^{12}\right.$ protons per pulse continuous operation, for example, is $1 \times 10^{20}$ protons per year).

3. Intensity limits due to airborne radiation are somewhat more restrictive. The existing air handling system will likely reach the limit for average release of airborne radiation of $100 \mathrm{Ci} / \mathrm{yr}$ at $1 \times 10^{20}$ protons per year. Increased intensity will require improved air handling. 


\section{E. Electron Cooling}

Electron cooling appears to be the only beam cooling technique practical for accumulating antiprotons with the intensity and emittance required for $\mathrm{TeV} 33$. It can reduce emittance simultaneously in all three degrees of freedom at a rate practically independent of the antiproton intensity. However, because the rate is inversely proportional to the cube of the emittance, stochastic cooling ideally complements it for pre-cooling the broad phase space distribution from the production target into one narrow enough for rapid electron cooling. In the TeV33 scenario, the Accumulator of the present Antiproton Source is adapted to cool a small stack quickly for injection into the Recycler at a rate somewhere between three times per minute and twice per hour. The Recycler accumulates antiprotons during the store, re-cooling the antiprotons recovered from the previous store and stacking the additional needed for the next store. The reference design brought to Snowmass '96 specifies a $66 \mathrm{~m}$ cooling region with discrete electron focusing elements every $2 \mathrm{~m}$. The 4.3 $\mathrm{MeV}, 2 \mathrm{~A}$ electron beam is produced by a Pelletron-type electrostatic accelerator* operating with high efficiency charge recovery. Because the capacity of the Pelletron charging system is a few hundred microamperes at most, attaining $2 \mathrm{~A}$ electron current requires that the maximum loss fraction be $10^{-4}$. This is one of several technical challenges in the present scheme which require extension of the existing technology.

The general parameters for a system based on a $4 \mathrm{MV}$ electrostatic accelerator are given in Table II. The underlying concept has been developing since the mid '80's with work by Fermilab, U. Wisconsin, and Indiana U.[16,17] An attempt by U. Wisconsin and Fermilab in the late '80's to demonstrate the required efficiency of charge recovery only achieved an electron current of $100 \mathrm{~mA}$. An improved version of this test is being set up at National Electrostatics Corp. as part of Fermilab's development effort. Another important aspect of the baseline plan currently being approached experimentally is the beam optics and dynamics of the long transport and periodic cooling region.

The Recycler receives newly produced antiprotons from the Accumulator frequently during a store, and, just before unloading its cooled stack into the Tevatron for a new store, it must accept about half a stack's worth of antiprotons from the Tevatron. These antiprotons have approximately twice the transverse emittance and perhaps three times the longitudinal emittance of beam from the Accumulator. While beam is being delivered from the Accumulator, electron cooling serves to reduce the stack momentum spread so that batch after batch can be fit into the longitudinal acceptance almost indefinitely.

\footnotetext{
*Pelletron is the trade name for electrostatic generators manufactured by National Electrostatics Corporation, Middleton WI. The main difference between a Pelletron and a Van de Graaff machines is the charging system, which uses chains of cylindrical metal pellets articulated by nylon connecting links.
}

From Table II parameters one can evaluate the maximum longitudinal drag rate

$$
\begin{aligned}
F_{d} & =\frac{4 \eta I\left(r_{e} m c^{2}\right)^{2} \Lambda}{\gamma_{r} e k T_{\perp} a^{2}} \\
& \cong 0.005 \frac{\eta I}{\gamma_{r} a^{2}} \mathrm{MeV} / \mathrm{sec}
\end{aligned}
$$

where $I$ is the electron current in A, $\eta$ is the fraction of the ring circumference occupied by electron beam, $r_{e}$ is the classical electron radius, $m c^{2}$ is the electron mass, $\gamma_{r}$ is the relativistic energy factor, $\mathrm{e}$ is the electron charge, $k$ is Boltzmann's constant, $T_{\perp}$ is the electron beam transverse temperature, $a$ is the electron beam radius in $\mathrm{m}$, and $\Lambda$ is the coulomb logarithm. The simpler second expression results from taking typical values $\Lambda=10, T_{\perp}=1200{ }^{\circ} \mathrm{K}$. The parameters from Table II can be used to set a lower bound on the time between Accumulator batches by dividing the calculated drag rate by the momentum spread in the Recycler after debunching. This is the quantity entered as $t_{\|}$in Table II for both accumulating and recycling conditions.

For the recycled antiprotons the transverse emittance determines the required cooling time. A satisfactory estimate for this time can be written from a simple model of the cooling:

$$
\begin{aligned}
t_{c} & =\frac{\beta_{r} \gamma_{r}^{2} a^{2} e \varepsilon_{\perp}^{3}}{12 \pi^{3} \Lambda r_{p} r_{e} \eta I x^{3}} \\
& \cong 3.087 \times 10^{11} \frac{\beta_{r} \gamma_{r}^{2} a^{2}}{\eta I}\left(\frac{\varepsilon_{\perp}}{\pi x}\right)^{3},
\end{aligned}
$$

where $\varepsilon_{\perp}$ is the normalized transverse emittance in m-rad, $r_{p}$ is the classical proton radius in $\mathrm{m}$, and $x$ is the antiproton beam radius in $\mathrm{m}$. This time is also given in Table II for accumulating and recycling conditions.

The consideration of electron cooling at Snowmass followed two somewhat independent lines, viz., critical examination of the baseline scheme and a study of alternatives employing electrons re-circulated for a few hundred turns in a modified betatron or storage ring supplied by an induction Linac. The first category involved review of the underlying assumptions, present parameter choices, and priorities for calculations and development activities. The second line involved an examination of the potential benefits of a stored electron beam and a first look at the hardware.

No sudden advance or radical insight was obtained regarding the baseline scheme. However, consideration of the role of the betatron and synchrotron oscillations of the antiprotons led to changing the electron beam radius to $2 \mathrm{~cm}$ from $1 \mathrm{~cm}$, an expectation of slightly faster cooling, and a priority for a full six-dimensional numerical model. We noted that, because the most effective transverse cooling comes at the extreme displacements of the betatron oscillation where the proton and electron velocities are closest, a large penalty in cooling of the recycled antiprotons is incurred for electron beam radius less 
Table II. Beam and system parameters for electron cooling in the Recycler

\begin{tabular}{lrl}
\hline \hline Electron energy & 4.87 & $\mathrm{MeV}$ \\
antiproton energy & 8.94 & $\mathrm{GeV}$ \\
Lorentz beta & 0.994 & \\
Lorentz gamma & 9.53 & \\
Antiproton $\varepsilon_{\perp}(6 \sigma$, normalized) & & \\
$\quad$ Stacking & $9.5 \pi$ & $\mathrm{mm}-\mathrm{mrad}$ \\
$\quad 20 \pi$ & $\mathrm{mm}-\mathrm{mrad}$ \\
$\quad$ Recycling & \pm 2 & $\mathrm{MeV}$ \\
Antiproton energy spread $( \pm 2 \sigma)$ & \pm 9 & $\mathrm{MeV}$ \\
$\quad$ Stacking & & \\
$\quad$ Recycling & 0.014 & $\mathrm{~m}$ \\
Antiproton beam radius & 0.02 & $\mathrm{~m}$ \\
$\quad 0.02$ & $\mathrm{~m}$ \\
$\quad$ Stacking & 1.12 & $\mathrm{~mm}-\mathrm{mrad}$ \\
$\quad$ Recycling & 0.005 & $\mathrm{~m}$ \\
Electron beam radius & 1200 & ${ }^{\circ} \mathrm{K}$ \\
Electron $\varepsilon_{\perp}(\mathrm{rms})$ & 2 & $\mathrm{~A}$ \\
Cathode radius & \pm 60 & $\mathrm{eV}$ \\
Cathode temperature & 99.99 & $\%$ \\
Electron beam current & 66 & $\mathrm{~m}$ \\
Electron energy stability & 3319 & $\mathrm{~m}$ \\
Charge recovery efficiency & 200 & $\mathrm{~m}$ \\
Length of cooling section & & \\
Ring circumference & 71 & $\mathrm{sec}$ \\
Antiproton Courant-Snyder $\beta_{x}, \beta_{y}$ & 219 & $\mathrm{sec}$ \\
Characteristic cooling time $\left(t_{c}\right)$ & & \\
$\quad$ Stacking & 16 & $\mathrm{sec}$ \\
$\quad$ Recycling & 67 & $\mathrm{sec}$ \\
Longitudinal cooling time $\left(t_{\|}\right)$ & $2-180$ & $\mathrm{hr}{ }^{-1}$ \\
$\quad$ Stacking & 0.17 & $\mathrm{hr}{ }^{-1}$ \\
$\quad$ Recycling & $\sim 1$ & $\mathrm{nTorr}$ \\
Recycler injection frequency & $\sim 2$ & $\mathrm{mG}$ \\
$\quad$ Stacking & & \\
$\quad$ Recycling & & \\
Ring Vacuum & Maximum stray magnetic field & \\
\hline \hline
\end{tabular}

than the antiproton beam radius. The gain in stacking rate from the Accumulator for a $1 \mathrm{~cm}$ radius would only be advantageous for a marginal system. Although the conclusions are probably correct, we are not aware of a good sixdimensional numerical model to check and quantify them. Whatever the final scheme, such a model would have wide application in the development effort.

The basic ideas favoring the re-circulation of the electrons in a storage ring for several passes through the cooling section are, first, that the electron injection duty factor can be lowered to a level where charge recovery is no longer required and, second, that higher electron current is plausible on the basis of existing experience. Because the antiprotons heat the electrons in proportion to the number ratio $N_{\bar{p}} / N_{e}$ and the mass ratio $m_{\bar{p}} / m_{e}$, storage periods of several milliseconds would be reasonable even for $N_{\bar{p}} \approx N_{e}$. Also a good beam is needed at only a percent of the kA levels that have been obtained in modified betatrons and induction Linacs. However, it is not clear whether an electron beam of adequate quality can be established and maintained for 1000 turns because the space charge tune shift is large. A modified betatron or a ring fed from an induction Linac could be small enough to fit into the Main Injector tunnel yielding a considerable savings in civil construction and installation costs compared to the Pelletron. The possibility of a factor of ten greater electron current suggests the construction of a shorter, simpler cooling section. If the system had a cooling section length of $6 \mathrm{~m}$ instead of the $66 \mathrm{~m}$ of the baseline design, one could contemplate performance gains by replicating the original system to fill more of the available MI-30 straight section, thereby gaining the operational advantage of redundant systems.

Because the modified betatron in particular is fairly modest in scale, experimental investigation of its performance as an electron beam source for cooling is practical even on a fairly short time scale. Consider, for example, a machine with a 0.5 $\mu \mathrm{sec}$ circulation period, $3 \mathrm{~A}$ circulating beam, and a $8.33 \mathrm{msec}$ cycle consisting of $1.66 \mathrm{msec}$ acceleration, $5 \mathrm{msec}$ cooling period, and $1.66 \mathrm{msec}$ ramp-down. The accelerating requirement is $1.3 \mathrm{kV}$ per turn which is $2 \mathrm{~V}$-sec; this voltage can be provided by a flux change of $2 \mathrm{~Wb}$ in a single induction cell. The wall plug power for a device of this type is about $100 \mathrm{~kW}$.

A rapid cycling induction Linac could inject high current into a $4.3 \mathrm{MeV}$ storage ring at a repetition rate of $100 \mathrm{~Hz}$ or even up to tens of kHz. The gaps would be driven by separate solid state modulators similar in principle to klystron modulators working at $10 \mathrm{kV}$. This is more elaborate electronically than the betatron scheme but has properties that could prove important. A fast injection into the storage ring at full energy would mean that the electrons could be injected during the short ion clearing gap in the antiproton beam which would therefore see constant electron beam properties. The beam optics of the storage ring is more straight forward and could make achieving the necessary beam quality more practical.

The Snowmass discussions indicated that the hardware for either Linac or betatron is technically feasible. Some of the unanswered questions about beam optics and beam dynamics are the same for both. It was decided to build some simple experimental devices to get an early assessment of the potential and the problems with the electron storage concept. The possibility of producing a compact device which could be both less expensive and more quickly installed encourages serious consideration of the storage idea despite somewhat greater uncertainty about its viability. This is a basically new proposal and has not been worked out in the same detail as the electrostatic accelerator approach. It seems sufficiently promising however, to justify some diversion of effort to establish the reality of the prospective advantages.

\section{CONCLUSIONS}

A number of $\mathrm{TeV} 33$ beam physics issues have been considered. A cursory look at possible initial store parameters 
has been completed and detailed beam-beam tune shift calculations have been performed. A strategy for increasing the antiproton flux has been outlined.

The beam-beam interaction issues need to be studied in more detail as outlined at the end of section IIB. Once this study has been completed, a more realistic study of initial store parameters can be made. The slip stacking simulations need to be improved to include coherent effects. We need to measure beam loading and the effectiveness of beam loading compensation on the Main Injector rf cavities (currently installed in the Main Ring). We need a more detailed understanding of the Antiproton Source aperture limitations and better alignment techniques need to be devised and tested. Significant progress must be made on beam cooling techniques. Despite the large amount of work remaining to be done, the possibility of an inexpensive program to achieve luminosities in the neighborhood of $1 \times 10^{33} \mathrm{~cm}^{-2} \mathrm{sec}^{-1}$ continues to be promising.

\section{ACKNOWLEDGMENTS}

This work was supported by the U.S. Department of Energy under contracts DE-AC02-76CH030000 and DE-AC03-76SF00515.

\section{REFERENCES}

[1] The TeV2000 committee report (unpublished), but currently available on the World Wide Web site http://www-theory.fnal.gov/tev33.ps.

[2] D. Amidei and R. Brock, ed., FERMILAB-Pub-96/082, 1996 (unpublished).

[3] D.A. Finley, in Proc. of the 1989 IEEE Particle Accelerator Conference, p. 1834.

[4] R.H. Siemann, submitted to this conference.

[5] J.A. Johnstone, "Report to the Low Beta Study Group I," Sept. 29, 1994 (unpublished).

[6] S.D. Holmes, et al., FERMILAB-TM-1920, 1994 (unpublished).

[7] B.W. Montague, CERN/ISR-GS/75-36, 1975, (unpublished).

[8] Shekhar Shukla, submitted to this conference.

[9] F. Bieniosek, M. Church, G. Jackson, S. O'Day, and R. Pasquinelli, to be published in the Proc. of the 5th European Particle Accelerator Conference, Barcelona, 1996.

[10] M. Church and E. Harms, private communication.

[11] S.C. O'Day, F.M. Bieniosek, Nucl. Instr, and Meth. A343, 343 (1994).

[12] G.I. Silvestrov, in AIP Conference Proceedings 372, Beam Dynamics and Technology Issues for $\mu^{+} \mu^{-}$ Colliders, 1995.

[13] F. Bieniosek, K. Anderson, and K. Fullett in Proc. IEEE/APS Particle Accelerator Conference, Dallas, 1995.
[14] F.M. Bieniosek, Fermilab Report No. Pbar-Note-561, 1996 (unpublished).

[15] D. Cossairt, Fermilab Environmental Protection Note 8, 1994 (unpublished).

[16] D.J. Larson, Ph.D. thesis, U. of Wisconsin (1986)

[17] MEB E-Cool Design Report 1992, Indiana U. Cyclotron Facility, 1992. 